\title{
Validation of a new food frequency questionnaire for measurement of dietary $n-3$ long chain fatty acids using the method of Triads
}

\author{
J. Lanigan ${ }^{1}$, S. Low ${ }^{1}$, M. Kokoreli ${ }^{1}$, K. Northstone ${ }^{2}$ and A Singhal ${ }^{1}$ \\ ${ }^{1}$ Childhood Nutrition Research Centre, University College London Institute of Child Health, 30 Guilford Street, London, \\ WCIN 1 EH and ${ }^{2}$ School of Social and Community Medicine, University of Bristol, UK
}

Long chain $n$-3 polyunsaturated fatty acids (n-3 LCPUFA) docosahexaenoic acid (DHA: 22:6 n-3) and eicosapentaenoic acid (EPA: 20:5 $n-3)$, present in high concentrations in certain types of fish, are reported to have benefits for cardiovascular disease (CVD). However, currently there is no validated instrument to measure dietary $n$-3 LCPUFA in the UK. A generalised food frequency questionnaire (FFQ), such as that used in the European Prospective Investigation of Cancer (EPIC) ${ }^{(1)}$, may estimate intake inaccurately because foods high in $n$ 3 LCPUFA are grouped together. The aim of this study was to assess whether a new FFQ which separated foods high in $n$-3 LCPUFA was a valid and reliable method for assessing $n-3$ intake in a group of young, healthy UK adults.

Participants $(n=78)$ in a randomised controlled trial investigating the effects of $n-3$ LCPUFA supplementation on CVD risk factors completed a new questionnaire (FishFQ), adapted from the EPIC FFQ, designed to collect dietary data on foods with the highest concentration of $n-3$ LCPUFA. The FishFQ comprised 31 food items and allowed separation of fish into white and oily, and discriminated between canned and fresh fish. The questionnaire was self administered after instructions were given by trained researchers.

Relative validity of the FishFQ was assessed using the method of triads which uses triangular comparisons between a dietary assessment method under test (FishFQ), a reference method (multiple 24 hour dietary recalls) and a biochemical marker (red cell membrane fatty acids) to compute validity coefficients $(\mathrm{VCs})^{(2)}$. Reliability was evaluated with Pearson's correlation coefficient at 4 month re-test interval.

VCs were similar for dietary assessment methods and biomarkers of $n$-3 LCPUFA intake. Both dietary assessment methods found higher VCs for DHA whereas for the biomarker a higher VC was found for EPA. Reliability was high for both fatty acids: $r 74$ and $r 55$ for DHA and EPA respectively.

\begin{tabular}{|c|c|c|c|c|c|c|c|c|c|}
\hline & \multicolumn{6}{|c|}{ Validity coefficients } & \multicolumn{3}{|c|}{ Range of Validity coefficients $\dagger$} \\
\hline & $P Q T$ & $95 \% \mathrm{CI}$ & $P$ PRT & $95 \% \mathrm{CI}$ & PMT & $95 \% \mathrm{CI}$ & PQT & $P$ PRT & PMT \\
\hline DHA & 0.54 & $(0.22,0.92)$ & 0.65 & $(0.32,1.28)$ & 0.63 & $(0.29,1.08)$ & $0.32-0.59$ & $0.42-0.71$ & $0.32-0.54$ \\
\hline EPA & 0.48 & $(0.26,0.76)$ & 0.63 & $0.33,1.00)$ & 0.80 & $0.48,1.44)$ & $0.47-0.61$ & $0.53-0.69$ & $0.47-0.78$ \\
\hline
\end{tabular}

† The lower limit for the FishFQ and the biomarker is the correlation between the Fish FQ and the biomarker, and the lower limit for the 24-HR is the correlation between the biomarker and the 24-HR. The upper limit is calculated by the method of triads. $p \mathrm{QT}$, validity coefficient of the FishFQ, $p$ RT, validity coefficient of the $24-\mathrm{H}$ recall, $p$ MT, validity coefficient of the biomarker. All data natural log transformed. 5\% confidence intervals computed from 200 Bootstrap samples.

Validity was comparable to findings from other studies which report VC's in the range of $0.22-0.60$ for fatty acids. Reliability was also high compared with previous studies involving FFQ's. Overall VC's for the FishFQ were high suggesting it is a valid method for dietary assessment of $n-3$ LCPUFA.

1. Bingham SA, Welch AA, McTaggart A, Mulligan AA, Runswick SA, Luben R et al. (2001) Nutritional methods in the European Prospective Investigation of Cancer in Norfolk. Public Health Nutr 4(3): 847-58.

2. Ocke MC \& Kaaks RJ (1997) Biochemical markers as additional measurements in dietary validity studies: application of the method of triads with examples from the European Prospective Investigation into Cancer and Nutrition. Am J Clin Nutr 65(4 Suppl): 1240S-5S. 